\title{
PA-037 ANTIBODY RESPONSE TO SEVERAL MALARIA ANTIGENS IS ASSOCIATED WITH PROTECTION FROM SEVERE MALARIA IN UGANDAN CHILDREN
}

Brenda Okech, ${ }^{1}$ Thomas Egwang, ${ }^{2}$ Patrick Corran, ${ }^{3}$ Eleanor Riley ${ }^{3}$. 'Makerere University, Uganda; ${ }^{2}$ Med Biotech Laboratories, Uganda; ${ }^{3}$ LSHTM, United Kingdom

10.1136/bmjgh-2016-000260.73

Background Malaria is one of the leading causes of morbidity. Both natural infection and irradiated sporozoites have yielded partial and sterile immunity and contain multiple antigens so a malaria vaccine with multiple antigens may be protective. Our hypothesis is that responses to several malaria antigens may protect against severe malaria. Currently various candidate vaccines are being tested and are in various stages. This study is from a case control study conducted in Apac district in Northern Uganda.

Methods Children with severe and complicated malaria were compared with those having uncomplicated malaria in a matched case-control study. Antibody titers in serum samples of children were assessed by ELISA and parasitaemia was quantified by microscopy.

Results When all children who had antibodies to at least 4 of the antigens tested were compared to children who responded to less than 4 of the antigens, multiple responders were significantly more prevalent in the mild malaria group. For all the antibodies studied, there was a tendency for the children with mild malaria to have higher OD levels than the children with severe malaria. Children who were responders to AMA1, $P$. falciparum lysate, SE36, MSP1 42, GPI, MSP2-2FC or MSP23D7 were significantly more likely to be in the mild malaria group.

Conclusions There were significant differences between children with severe and mild malaria for antibodies. Higher levels of these antibodies were associated with protection from severe malaria disease and from high parasitaemia. Our data suggest a role for multiple blood stage antigen vaccines and denatured toxin vaccine supports the development of multiple component vaccines. 